\title{
Pastoral care in hospitals: a literature review
}

\author{
Tullio Proserpio', Claudia Piccinelli², and Carlo Alfredo Clerici² \\ 'Pastoral Care Unit, IRCCS Foundation National Cancer Institute of Milan; '2Department of Biomolecular \\ Sciences and Biotechnology, Psychology Section, University School of Medicine, Milan, Italy
}

\section{ABSTRACT}

Aims and background. This literature review investigates the potential contribution of the pastoral care provided in hospitals by hospital chaplains, as part of an integrated view of patient care, particularly in institutions dealing with severe disease.

Methods and study design. A search was conducted in the Medline database covering the last 10 years.

Results. Ninety-eight articles were considered concerning the modern hospital chaplains' relationships and the principal procedures and practices associated with their roles, i.e., their relations with the scientific world, with other religious figures in the community, with other faiths and religious confessions, with other public health professionals and operators, with colleagues in professional associations and training activities, and with the hospital organization as a whole, as well as their patient assessment activities and the spiritual-religious support they provide, also for the patients' families.

Conclusions. Improvements are needed on several fronts to professionalize the pastoral care provided in hospitals and modernize the figure of the hospital chaplain. These improvements include better relations between modern chaplains and the hospital organization and scientific world; more focus on a scientific approach to their activities and on evaluating the efficacy of pastoral care activities; greater clarity in the definition of the goals, methods and procedures; the design of protocols and a stance on important ethical issues; respect for the various faiths, different cultures and both religious and nonreligious or secularized customs; greater involvement in the multidisciplinary patient care teams, of which the hospital chaplains are an integral part; stronger integration with public health operators and cooperation with the psychosocial professions; specific training on pastoral care and professional certification of chaplains; and the development of shared ethical codes for the profession.

\section{Introduction}

Humanizing the medical care afforded by the most advanced public health services implicates integrating the biological, psychological and social aspects of the patient's needs, which also includes the patient's spiritual and/or religious inclinations and background.

Emerging phenomena, such as the increasingly multicultural nature of modern society and the need to base patient care practices on scientific evidence, make it necessary to seek a better understanding of the potential contribution of spiritual care in the hospital setting, particularly in departments and institutions where severe diseases are treated.

The present study consisted of a review of the scientific medical literature relating to the activities of hospital chaplains, focusing on the relational and organizational aspects and working practices.

\section{Materials and methods}

The material used in this review consisted of articles identified in the Medline database published over the last 10 years. The key words used for the search were as fol-

Key words: spiritual care, chaplain, hospital chaplaincy, pastoral care, biopsycho-socio-spiritual integration.

Correspondence to: Don Tullio Proserpio, Cappellania Ospedaliera, Fondazione IRCCS Istituto Nazionale Tumori, Via Venezian 1, 20133 Milan, Italy. Tel +39-02-23902323;

e-mail

tullio.proserpio@istitutotumori.mi.it

Received November 29, 2010; accepted March 11, 2011. 
lows: spiritual care, chaplain, hospital chaplain, and pastoral care. Among the 288 references identified, 98 articles (all written in the English language) were considered of interest for the purposes of this review.

The 98 articles selected were classified as qualitative or quantitative in nature. Only about 20 of them were quantitative studies, including between-group comparisons ${ }^{1-}$ 7 in which some, albeit elementary, statistical analyses were conducted; investigations conducted on different sample populations selected in various ways ${ }^{8-17}$; research on specific issues ${ }^{18-20}$; and literature reviews ${ }^{21,22}$. The vast majority of the articles were qualitative studies reporting descriptive data, sometimes in the form of case reports, letters to editors, editorials and study designs. The USA was the country most active in generating the publications considered (with 63 articles), followed by the UK (17 articles) and Australia (7 articles).

The content of the articles is discussed below, focusing on the aspects that seemed most significant considering the currently felt need to integrate spiritual and multicultural aspects in the patient treatment process.

\section{Results}

\section{Relationship with the scientific world}

More than a third of the articles concerned the efficacy and scientific nature of the service provided by hospital chaplains ${ }^{5,6,9-11,13,19,23-37,38}$. These studies generally emphasized the need to publish research, reviews and quality analyses with a view to documenting the effectiveness of the chaplains' activities and to measuring the results, validating the methods adopted, and assessing the quality of this service and the patients' satisfaction with the chaplains' work, in order to avoid their role being considered only a marginal aspect of the public health services.

\section{Exchanges and relations with other religious figures in the community}

In more than $90 \%$ of the articles examined, the figure of the spiritual care provider in hospitals that was considered in the study coincided with the hospital chaplain. One in 4 of the articles also referred to other religious figures as well as the chaplains appointed to provide pastoral care in hospitals, including local community priests and clergymen, and other ministers and religious figures $8,10,11,13,14,17,19,21,25,32,37,39-41$; this prompted considerations on the relationships between hospital chaplains and the other religious figures to which patients refer outside the hospital.

Hospital chaplains have relations with local religious leaders, as well as sharing the responsibilities and cooperating with colleagues at other healthcare institutions (e.g., between the hospital and the hospice, where these constitute different structures). The chaplains cooperate with the local religious community to facilitate the link to pastoral care within the public health organization, and they have good relations with the various local confessions. Where the chaplain is a Christian (as was usually the case, probably because of the cultural background of the journals considered), he acts as a link with the local clergy and interacts with laypeople willing to cooperate in this setting.

\section{Relationship with other faiths}

Spiritual-religious care is also discussed in terms of the multiplicity of faiths and religious confessions.

About 20 articles refer to different religious confes-

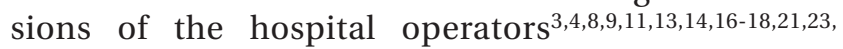
$24,29,31,37,39-44$, while only a handful of papers mention multilingual chaplains and multireligious chaplaincies $^{18,24,29,37,39,40,43}$.

Importance is attributed to respecting the diversity of gender, culture and faith, to ensuring religious freedom and providing the opportunity to conduct rituals that are important to patients. Many facets of these issues are discussed. Some authors propose general guidelines based on an interconfessional approach that provide for an assessment of patients' religious beliefs and customs (including matters of clothing, rituals, diet, etc.), the most appropriate places for practicing their faith, and the provision of interconfessional halls as well as traditional chapels as a focal point for providing pastoral care ${ }^{29,40,43}$.

\section{Relationship with other public health professionals and operators}

The literature considered discusses the relations between the chaplains and the members of the hospital staff and patient care teams on various hierarchical levels and from the individual, interdisciplinary, multidisciplinary, training/educational and operational standpoints.

In particular, several topics were recurrently covered, namely

- the chaplain's relations with nurses, which are discussed in $25 \%$ of the articles considered ${ }^{8,23,42,44,45}$;

- the fact that the more nurses are religious, the more they are likely to recommend that patients see the chaplain $^{1,23,42,44}$;

- the generally satisfactory relationship between physicians and chaplains ${ }^{16,23,46}$;

- aspects of the relations between physicians and chaplains $^{16,17,28,42,44,46,47}$;

- the religious convictions of physicians and how they interact with the chaplain ${ }^{1,16,17,44,46 ;}$

- physicians' training regarding their patients' spirituality concerns, and physicians' relations with chaplains ${ }^{12,16,27,28,30,46}$;

- aspects of the chaplains' relations with physicians are discussed in $34 \%$ of the articles considered;

- the chaplains' role as members of the patient care team is dealt with in $40 \%$ of the articles ${ }^{7,10,13,14,17,22-}$ $24,26,28,30,32,36,38-40,45-48$ 
The relationship between chaplains and nursing personnel was generally judged to be satisfactory.

\section{Procedures and practices}

\section{Informal or formal assessments, clinical files}

Approximately 1 in 3 of the articles examined referred to the identification or assessment of patient's preferences concerning religious support and/or the recording of this information in their clinical files ${ }^{5-8,11,13,14,17,18 \text {, }}$ $23,25,33,34,37,40,44,45,48$.

Since 1997 the provisions of the Joint Commission on Accreditation of Healthcare Organizations (JCAHO) have had a positive fallout in terms of global care focusing on the patient as a person. The chaplains' role has become more integrated in the multidisciplinary team and they are more involved in assessing patients, providing care and evaluating outcomes. Hospital stays have become shorter than in the past and chaplains have less time to deal with a patient's needs, their visits have become fewer and shorter, so it is more difficult for them to conduct spiritual assessments, recognize patients' hopes and fears, and the spiritual resources they can rely on, both in themselves and within their families and communities ${ }^{18}$.

\section{Description of the chaplain's activities: support extending to patients' families}

More than $30 \%$ of the articles mention chaplains providing support for patients' families $3,4,10,11,14,17,18,23,24,26$, 28,30,41,42,44,47,49.

A chaplain's work does not focus on the patient alone, but extends to the patient's family too with a view to improving the quality of life of the family system as a whole. In this sense, it is important to consider the problems that develop, for instance, when relatives refuse to tell patients the truth about their diagnosis, or when ethical issues arise in cases of therapeutic obstinacy, when relatives demand further treatments. Chaplains can help patients' families prepare themselves for the death of their relative, or help physicians give patients and their families bad news.

Spiritual care therefore also includes a patient's family, and this poses problems relating to the availability of resources, and the frequency and duration of the chaplain's visits to the patient's relatives.

Importance is attributed to the subject of the tools to use in assessing patients and the service to provide for patients' families, particularly as concerns families coping with bereavement and how long the service should be provided following the loss of a loved one ${ }^{18,41}$.

\section{Relationship with the hospital organization}

Regarding the relationship between the figure of the chaplain and the organization where he works $4,5,8,12,14$, $15,19,20,23,24,26,30,31,37,38,40,49$, some issues were frequently discussed:
- a demand for spirituality that is not met ${ }^{6,9,11,13,19,23,}$ 25,29,39,43,46;

- the limited resources available for spirituality ${ }^{8,11,46}$;

- the voluntary chaplain ${ }^{6,8,14,15,17,42,43}$;

- support for healthcare operators in ethical issues (resuscitation or feedback on pain control) ${ }^{4,10,13,14,17,20,26,30,37,40,47}$;

- part-time chaplains ${ }^{6,8,15,17,42}$;

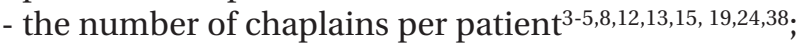

- the duration of the chaplain's visits ${ }^{3,4,42}$;

- the greater efficacy of official chaplains than voluntary chaplains in supporting staff dealing with the problem of whether to stop life-support systems ${ }^{14,17}$.

The fact that the agencies responsible for assessing public healthcare standards acknowledge the patient's spiritual needs means that chaplains must also comply with the requirements of quality standards and certification procedures. Chaplains have to understand the dynamics of the organization in which they operate.

Relationship with colleagues, professional associations and training

The training of chaplains and the certification of suitably trained chaplains are considered a priority because training and certification are seen as the fundamental tools for operating in highly complex occupational settings demanding a high level of expertise. Proper training is often identified as a solution to many of the problems encountered in the chaplain's work: $50 \%$ of the articles discuss the hospital chaplain's training $3,4,8-10,14$, $15,17,21-26,29-31,36-39,40,43,47,49$, and $37 \%$ of them refer to professional associations of hospital chaplains ${ }^{6,8,12-15,22-26,30-}$ $32,37,38,40,48$.

The modern chaplain is quite unlike the traditional concept of a voluntary service provided by a person with no specific training.

\section{Discussion and conclusions}

Several critical issues emerged from this literature review. One problem concerns the chaplains' approach to their work. The evidence-based scientific medical care setting in which the hospital chaplain operates tends to demand an assessment of the efficacy of all action taken, including pastoral care activities, focusing particularly on the patients' quality of life and the satisfaction of their needs.

There is evidence in the literature considered of a debate on whether chaplains should have a scientific approach or not: those opting against this claim that adopting a scientific approach to their activities interferes with the fundamental nature of pastoral care, leading to reductionism; those supporting the need for a more scientific approach feel that hospital chaplains operate within a medical care setting, in an environment that relies intrin- 
sically on scientific evidence, so they too cannot escape the need to demonstrate the efficacy of their actions. Taking a scientific perspective may help to clarify certain priority issues, for instance, how to integrate spirituality in health treatment; how to acquire more adequate research tools and assessment methods; how to validate the tools used to investigate a patient's spiritual needs and how well these needs are met; how to describe the nature of spiritual care and identify the biological mechanisms of action of spirituality on a person's health.

One of the solutions proposed to orient chaplains towards a more scientific approach concerns the training of future professionals and would involve enriching their training curricula, establishing broader-based research groups, and facilitating exchanges of opinions between colleagues on research issues.

On the whole, there is a move towards a pastoral care configured as an activity with clear objectives, a comprehensible methodology and shared procedures. Modern educational schemes will be needed to train religious operators also on issues relating to scientific research.

Ample space in the scientific literature is dedicated to the relationship between different cultures and religious faiths, a topical issue in our modern world characterized by migratory phenomena and globalization. This imposes the need to found spiritual debate on the opportunity to exchange views with everyone, irrespective of the confession to which they belong, to ensure freedom of religion and free access to religious support, and the need to avoid dangerous divisions and contrasts on the basis of belief. Some authors suggest to go beyond the traditional model of the chaplain concerned almost exclusively with the faithful of his own religion, and to develop a model capable of helping people oriented towards different faiths and cultures, and also towards beliefs founded not on religious grounds $^{9,39}$. In a multicultural society, mechanisms and protocols are needed to guarantee the multireligious and interconfessional nature of the spiritual care provided in hospitals, with the attention and respect contemplated, among other things, in the Nostra Aetate Declaration during the Second Vatican Council on the matter of the Catholic Church's relationships with nonChristian religions ${ }^{50}$.

Most of the literature examined concerns the chaplain's relationship with public health operators: in a modern perspective the chaplain is increasingly identified as part of the multidisciplinary team, but the chaplain's effective cooperation with the other professional figures in the hospital and his involvement in the interdisciplinary and multidisciplinary patient care team still meet with some obstacles. These include a limited awareness within the hospital setting of the specific training that hospital chaplains receive, which qualifies them to operate in this particular environment.

Improving the exchange of views between the chaplains and other public health operators could have a considerable benefit in terms of facilitating their cooperation, making sure that the know-how and methods on which spiritual care is founded are clear and comprehensible.

The referral to spiritual care is an important topic. There are differences between the various professions and between genders in terms of the number of patients referred to the chaplain by the different public health operators (nursing staff are more likely to be female and more religious than other public healthcare professionals, and they are more likely than physicians to refer patients to a chaplain).

It would clearly be useful for staff members to exchange their views on how and when patients should be referred to a chaplain to deal with their spiritual needs. It would be advisable to develop standard protocols for referring patients to the chaplain.

The topic of the limited numbers of patients referred for pastoral care by some public health figures is linked to the issue concerning the acknowledgment and consideration of the chaplain's role by other professionals and the directors responsible for the various hospital departments. Differences are reported in how department directors see the importance of the chaplain's role: many chief physicians of medical disciplines have less consideration for the role of the chaplain than the directors of other disciplines. Especially in hospitals with a limited number of beds, these physicians often see the chaplain's activities in traditional terms (to celebrate religious rites), while they rarely acknowledge the chaplain's role on a hierarchically higher level. For instance, they often do not know that hospital chaplains receive certified specific training and therefore underestimate their qualifications to provide pastoral care in this setting. The chaplain is rarely considered as part of the patient treatment team, or a person to include in any interdisciplinary exchange of views. A possible solution to this problem would be to facilitate the chaplain's communications with the other professionals and ensure that they provide clear and complete information on their expertise, training and role ${ }^{10}$.

The chaplain's job is not limited to providing spiritual care for patients and their families, but may extend to supporting the hospital staff in relation to spiritual issues that can affect the work of other public health operators.

The chaplain may also have a part to play in balancing questions of ethics and faith, or serving as an intermediary, or as a bridge in communications between physicians and their patients or the patients' relatives ${ }^{13}$. Chaplains should never substitute other healthcare professionals, interfere with the physicians, or invade the spaces occupied by other professional figures. It is essential that they never step over the boundaries with other disciplines: chaplains need to be able to clearly describe the range of their actions and respect the limits on their activities.

None of the articles examined took into consideration the relations between chaplains and psychologists, possi- 
bly because all the papers analyzed were drawn from publications in the medical sector. It was consequently difficult to see any clear distinction between the role of these two figures in articles that mention patients being referred to the chaplain for problems relating to the emotional sphere where no psychologist was available, or referrals motivated by symptoms of anxiety or depression. It might be useful in the future to delineate the relationship that exists between psychological care and spiritual care ${ }^{44}$.

Whether chaplains are part of the treatment team or not leads to the question of whether or not they are entitled to access a patient's clinical files and, if so, under what circumstances (to get to know the patient, to record information, to compile parallel documents, and so on).

There is some debate concerning the various aspects of patient assessment, the tools to use (more or less indepth questionnaires or informal records, that may or may not be attached to a patient's personal and social details), and who should conduct the assessment whether this should be the responsibility of the chaplain or other personnel. This topic is amply discussed in the literature considered, which raises questions of privacy, the right to confidentiality, and the patient's right to express their consent on the matter of spiritual care.

A hospital chaplain's main activities therefore depend on the public health setting in which they operate and may include identifying and assessing patients' needs (their spiritual, religious and cultural demands, the preferences dictated by their beliefs and religious customs) and their expectations in relation to various factors (e.g., depending on a patient's age and gender, whether they may expect a spontaneous visit from the chaplain or prefer this to be available on request, or they may simply want to be told how to request a visit from the chaplain should they feel the need). Reference is made to documents produced by the chaplain to communicate with the public, publications to illustrate the services available on matters of spiritual care, and general guidelines and protocols for operating in an interconfessional context. On the issue of patient assessment, some publications discuss whether the best timing for this activity is on admission or during the hospital stay, once a therapeutic alliance has been established, and which other professional figures should be involved in this assessment.

Modern hospitals that strive to provide solutions to the numerous, multifaceted demands of their patients may discover an additional resource in the figure of today's hospital chaplain. There are many variables to consider with a view to organizing a chaplaincy service adequately, including an in-depth analysis of the organization's objectives and mission (e.g., whether it includes intensive care units or hospices, or institutions dedicated exclusively to treating oncological diseases). The publications reviewed mention the importance of thoroughly examining the criteria for measuring productivity and the hospital's organization chart. In fact, modern administrations also demand that chaplains justify their operations. Importance is attributed to the sizing and structure of the chaplaincy, i.e., the ratio of chaplains to patients, the size of the hospital, the hospital's religious affiliation, the presence of a cancer ward, and the location of the hospital.

The chaplains' professional qualifications are important too, as are the contracts governing their employment as professionals, rather than relying on voluntary workers and consequently being able to impose fewer demands on their relevant training.

The scientific literature analyzed in this review provides evidence of the need for the hospital chaplain's role to combine traditional and modern working practices (ethical consulting, emotional support, the ability to listen, making sense of and giving meaning to disease, training activities) as part of their relations with the scientific world, the hospital organization and the various religious confessions.

The chaplain's work is documented differently in different countries. Most of the studies were published in the United States and the United Kingdom, while our search among journals with an impact factor in the medical sector identified no such studies on the situation in Italy, which would be worth investigating. It seems feasible to claim that a scientific approach, suitable training, and standards regulating both the ethical concerns and the working procedures are the right building blocks for the modernization and professionalization of the figure of the hospital chaplain.

Examples of the chaplain's integration in the international hospital scenario, and in other complex settings too (such as - oddly enough - the United States Army, where there is even a Field Manual on the provision of religious support), offer important prospects for further analysis.

\section{References}

1. Flannelly KJ, Galek K: Discipline and sex differences in religiosity and spirituality among health care professionals. Psychol Rep, 99: 803-804, 2006.

2. Handzo GF, Flannelly KJ, Kudler T, Fogg SL, Harding SR, Hasan YH, Ross AM, Taylor BE: What do chaplains really do? II. Interventions in the New York Chaplaincy Study. J Health Care Chaplain, 14: 39-56, 2008.

3. Handzo GF, Flannelly KJ, Murphy KM, Bauman JP, Oettinger M, Goodell E, Hasan YH, Barrie DP, Jacobs MR: What do chaplains really do? I. Visitation in the New York Chaplaincy Study. J Health Care Chaplain, 14: 20-38, 2008.

4. Vanderwerker LC, Handzo GF, Fogg SL, Overvold JA: Selected findings from the "New York" and the "Metropolitan" chaplaincy studies: a 10-year comparison of chaplaincy in the New York City area. J Health Care Chaplain, 15: 13-24, 2008.

5. King DE, Platz E: Addressing the spiritual concerns of patients in the non-intensive care setting. South Med J, 96: 321-322, 2003.

6. Kernohan WG, Waldron M, McAfee C, Cochrane B, Hasson F: An evidence base for a palliative care chaplaincy service in Northern Ireland. Palliat Med, 21: 519-525, 2007. 
7. Finlay E, Lu HL, Henderson H, O’Dwyer PJ, Casarett DJ: Do phase 1 patients have greater needs for palliative care compared with other cancer patients? Cancer, 115: 446-453, 2009.

8. Wright MC: Chaplaincy in hospice and hospital: findings from a survey in England and Wales. Palliat Med, 15: 229242, 2001.

9. Sheikh A, Gatrad AR, Sheikh U, Panesar SS, Shafi S: Hospital chaplaincy units show bias towards Christianity. BMJ, 329: 626, 2004.

10. Flannelly KJ, Galek K, Bucchino J, Handzo GF, Tannenbaum HP: Department directors' perceptions of the roles and functions of hospital chaplains: a national survey. Hosp Top, 83: 19-27, 2005.

11. Tzeng H, Yin C: Demands for religious care in the Taiwanese health system. Nurs Ethics, 13: 163-179, 2006.

12. Cadge W, Freese J, Christakis NA: The provision of hospital chaplaincy in the United States: a national overview. South Med J, 101: 626-630, 2008.

13. Piderman KM, Marek DV, Jenkins SM, Johnson ME, Buryska JF, Mueller PS: Patients' expectations of hospital chaplains. Mayo Clin Proc, 83: 58-65, 2008.

14. Carey LB, Newell CJ: Withdrawal of life support and chaplaincy in Australia. Crit Care Resusc, 9: 34-39, 2007.

15. VandeCreek L, Siegel K, Gorey E, Brown S, Toperzer R: How many chaplains per 100 inpatients? Benchmarks of health care chaplaincy departments. J Pastoral Care, 55: 289-301, 2001.

16. Fitchett G, Rasinski K, Cadge W, Curlin FA: Physicians' experience and satisfaction with chaplains: a national survey. Arch Intern Med, 169: 1808-1810, 2009.

17. Carey LB, Newell CJ: Chaplaincy and resuscitation. Resuscitation, 75: 12-22, 2007.

18. Beardsley C: 'In need of further tuning': Using a US patient satisfaction with chaplaincy instrument in a UK multifaith setting, including the bereaved. Clin Med, 9: 53-58, 2009.

19. Flannelly KJ, Galek K, Handzo GF: To what extent are the spiritual needs of hospital patients being met? Int J Psychiatry Med, 35: 319-323, 2005.

20. Goy ER, Carlson B, Simopoulos N, Jackson A, Ganzini L: Determinants of Oregon hospice chaplains' views on physician-assisted suicide. J Palliat Care, 22: 83-90, 2006.

21. Flannelly KJ, Weaver AJ, Smith WJ, Oppenheimer JE: A systematic review on chaplains and community-based clergy in three palliative care journals: 1990-1999. Am J Hosp Palliat Care, 20: 263-268, 2003.

22. Ford T, Tartaglia A: The development, status, and future of healthcare chaplaincy. South Med J, 99: 675-679, 2006.

23. McClung E, Grossoehme DH, Jacobson AF: Collaborating with chaplains to meet spiritual needs. Medsurg Nurs, 15: 147-156, 2006.

24. Handzo G, Wintz S: Professional chaplaincy: establishing a hospital-based department. Healthc Exec, 21: 38-39, 2006.

25. Self G: Between mandate and patient need. Chaplains at a Canadian facility felt challenged in adapting themselves to briefer hospital stays. Health Prog, 81: 32-36, 2000.

26. Hughes B, Whitmer M, Hurst S: Innovative solutions: a plurality of vision - integrating the chaplain into the critical care unit. Dimens Crit Care Nurs, 26: 91-95, 2007.

27. Austin LJ: True evidence-based care. Hosp Health Netw, 77: $10,2003$.

28. Poehlman K: Spirituality and medicine. Health Prog, 85: $54-55,2004$.

29. Gatrad AR, Brown E, Sheikh A: Developing multi-faith chaplaincy. Arch Dis Child, 89: 504-505, 2004.
30. Feldbush MW: Healthcare chaplaincy: taking a look at the new model. South Med J, 101: 580, 2008.

31. Orton MJ: Transforming chaplaincy: the emergence of a healthcare pastoral care for a post-modern world. J Health Care Chaplain, 15: 114-131, 2008.

32. Lyndes KA, Fitchett G, Thomason CL, Berlinger N, Jacobs MR: Chaplains and quality improvement: can we make our case by improving our care? J Health Care Chaplain, 15: 6579, 2008.

33. Rosendahl J, Tigges-Limmer K, Gummert J, Dziewas R, Albes JM, Strauss B: Bypass surgery with psychological and spiritual support (the By.pass Study): study design and research methods. Am Heart J, 158: 8-14, 2009.

34. Weaver AJ, Flannelly KJ, Liu C: Chaplaincy research: its value, its quality, and its future. J Health Care Chaplain, 14: 3$19,2008$.

35. Weaver AJ: Mission to enhance the quality and quantity of chaplaincy research. preface. J Health Care Chaplain, 14: 12, 2008.

36. Berlinger N: The nature of chaplaincy and the goals of QI: patient-centered care as professional responsibility. Hastings Cent Rep, 38: 30-33, 2008.

37. Handzo GF: Best practices in professional pastoral care. South Med J, 99: 663-664, 2006.

38. Lambert JM, Connelly MD: Revitalizing spiritual care. A Catholic system develops a way to replenish and expand its ranks of chaplains. Health Prog, 88: 36-40, 2007.

39. Sutherland M: The challenge is to develop a truly multifaith approach, not plural monofaithism. Ment Health Today, April: 20, 2006.

40. Le Doux Sakurai M: The challenge and heart of chaplaincy. Recent decades have brought a host of changes to the health care chaplain's role. Health Prog, 84: 26-28, 56, 2003.

41. Walter T: Hospices and rituals after death: a survey of British hospice chaplains. Int J Palliat Nurs, 9: 80-85, 2003.

42. Flannelly KJ, Weaver AJ, Handzo GF: A three-year study of chaplains' professional activities at Memorial Sloan-Kettering Cancer Center in New York City. Psychooncology, 12: 760-768, 2003.

43. Gatrad AR, Sadiq R, Sheikh A: Multifaith chaplaincy. Lancet, 362: 748, 2003.

44. Vanderwerker LC, Flannelly KJ, Galek K, Harding SR, Handzo GF, Oettinger M, Bauman JP: What do chaplains really do? III. Referrals in the New York Chaplaincy Study. J Health Care Chaplain, 14: 57-73, 2008.

45. Ekedahl M, Wengström Y: Coping processes in a multidisciplinary healthcare team - a comparison of nurses in cancer care and hospital chaplains. Eur J Cancer Care (Engl), 17: 42-48, 2008.

46. Ferrell B: Meeting spiritual needs: what is an oncologist to do? J Clin Oncol, 25: 467-468, 2007.

47. Carey LB, Newell CJ, Rumbold B: Pain control and chaplaincy in Australia. J Pain Symptom Manage, 32: 589-601, 2006.

48. Loewy RS, Loewy EH: Healthcare and the hospital chaplain. MedGenMed, 9: 53, 2007.

49. European Network of Health Care Chaplaincy: Standards for healthcare chaplaincy in Europe: South Med J, 99: 682683, 2006.

50. Declaration on the relation of the Roman Catholic Church to non-Christian religions Nostra Aetate, proclaimed by Pope Paul VI on October 28, 1965. Available at http://www.vatican.va/archive/hist_councils/ii_vatican_c ouncil/documents/vat-ii_decl_19651028_nostraaetate_en.html 